\title{
Routing in Wireless Ad Hoc Networks by Analogy to Electrostatic Theory
}

\author{
Mehdi Kalantari and Mark Shayman \\ Department of Electrical and Computer Engineering \\ University of Maryland, College Park, MD 20742 \\ \{mehkalan, shayman\}@eng.umd.edu
}

\begin{abstract}
In this paper we introduce a novel approach for the routing problem in wireless ad hoc networks. Our approach is based on the analogy of the routing problem to the distribution of electric field in a physical media with a given density of charges. We show that the throughput can be significantly increased by choosing routes in such a way that the traffic is spread as uniformly as possible throughout the network. Achieving this uniform spreading requires solution of a set of partial differential equations similar to Maxwell's equations in the electrostatic theory. While the main focus in the paper is on the case in which many sources communicate with a single destination, extension to the case of multiple destinations is also described.
\end{abstract}

\section{INTRODUCTION}

Wireless ad hoc networks have received much attention in recent years. These networks have no infrastructure and the design of network protocols should be such that the nodes can be able to find the proper routes to their destinations and identify a good network topology. In these networks, nodes relay the packets of the other nodes toward the desired destination. A random access scheme is generally used as the MAC layer for these networks, but since the transmission range of the nodes is limited, a collision only happens when two simultaneously transmitting nodes are close enough to each other. When we have several nodes competing for the resources, it is logical to make the competing nodes as apart from each other as possible; the main idea of our work is to route different flows in the network in a way that the network load is as uniform as possible to reduce the rate of collisions and achieve a higher throughput in the network. We will define an abstract concept called load based on the location of sources and destinations and the amount of bandwidth needed by each flow. We develop the mathematical formulation of the load, and then we explain how it can be used to solve the routing problem. It will be shown that the optimal distribution of the load in the network leads us to a set of partial differential equations similar to those governing distribution of electric field in a physical media in the context of electrostatic theory.

Link state and distance vector are the conventional methods for the purpose of routing in the data networks [5]. Modifications to these methods have been done to use them in wireless ad hoc networks. Fisheye State Routing [6] [7] and Ad Hoc On Demand Distance Vector [8] are among the proposed protocols. A good survey on the routing schemes of wireless ad hoc networks can be found in [4].

There is another class of routing protocols that make use

This work was partially supported by AFOSR under contract F496200210217. of the geographical information of the wireless nodes. In Location Aided Routing [9] the routes are established on demand from the source node. The location information is used to limit the nodes that perform the flooding mechanism for route discovery. Distance Routing Effect Algorithm for Mobility [11] also uses the location information for routing. In this approach each node keeps a location table that stores and updates the location of all nodes in the network. Each node periodically sends a message to the other nodes and advertises its location information. The frequency of updating depends on the distance of the nodes and the rate of mobility. The location information is used to relay packets through the nodes that are in the direction of the destination.

We start with a network of wireless nodes with no mobility, known traffic demands for each sources-destination pair and known physical location of sources and destinations. Furthermore, we assume this information is available at a central route server that can compute and assign the routes to the different source-destination pairs. These assumptions may be reasonable for sensor networks. For other applications, these assumptions are somewhat restrictive, but we use them for this initial work on electrostatic routing, and we expect to be able to relax them in future work. For example, location of sources and destinations and the demand information might be advertised through the network by using some flooding mechanism, leading to distributed computation of routes.

When we have a request for communication at a source node, we can consider the source node as a source of load. Since the source is away from its destination, there is a need for communication resources in the space between the source and the destination. Generally the intermediate nodes in the network are needed to contribute transmission of data, so the demand of a source-destination pair imposes some load in the intermediate nodes as well; the way this load is distributed in the network depends on how the data is routed from the source to the destination geographically. One feature of our work is that we define the load as a function of space and not as a function of node.

We introduce a novel approach to solve the routing problem in the network by formulating it as an optimization problem. We make use of the concept of vector fields to define the load at every place of the network and show that this vector field will be conservative under certain assumptions. By using this conservative vector field we define a very powerful tool for routing by writing it as the gradient of a scalar potential function. The routing of packets to each destination is done based on the value of this potential function on each node 
and its value on the neighboring nodes. In this paper our primary focus is on the many-to-one scenario in which many sources want to send their data toward a single destination. Our results show considerable improvements in the network performance in terms of throughput for this case. Then we generalize our approach to the many to many scenario in which every arbitrary pair of nodes can communicate with each other.

The remainder of the paper is organized as follows. In Section II, we define the concept of load and the mathematical framework of using vector fields to formulate it and clarify the main concept by an example. In Section III we generalize the definition of the load vector field given in Section II and state the optimization problem for uniform load distribution. We solve the problem for many-to-one communication first, and then we extend our approach to the general case. In Section IV, we will define an example scenario and show the numerical results of solving the partial differential equations that give the optimal distribution of the load in the network. This section also contains the results of some simulation experiments to evaluate our approach.

\section{Mathematical Formulation}

Consider a network with $N$ wireless nodes that can communicate with each other through radio links. The nodes are randomly placed in a region $A$ in the plane. Assume there are $M$ source-destination pairs, denoted $s_{1}, \ldots, s_{M}$. Sourcedestination pair $s_{i}$ has a bandwidth demand which we refer to as its weight and denote by $W_{i}$. Suppose that one or more paths in the plane are chosen for each $s_{i}$. Each path starts at the source node and ends at the destination node of $s_{i}$. The weight $W_{i}$ is partitioned into amounts that are assigned to the paths. The weight assigned to a particular path indicates the amount of demand that is desired to follow that path. It should be noted that the chosen paths are not constrained by the location of intermediate nodes. Instead, the paths are 'abstract' paths in the plane that represent desired paths for the transit of packets. For communication to occur, each abstract path must be approximated by an actual path consisting of a piecewise linear multihop path connecting the source and destination through a sequence of of intermediate nodes.

Given a set of weighted (abstract) paths for each sourcedestination pair, we define a vector field on $A$ which we refer to as the load density vector field and denote by $\vec{D}$. $\vec{D}$ represents the flux density of the weighted paths for the source-destination pairs. Given a point $(x, y) \in A$, we choose a small area element at $(x, y)$. For each path that intersects $S$, we take the tangent vector to the path and scale it so it has magnitude equal to the weight of the path. Adding up these scaled tangent vectors, dividing by the area of $S$, and letting the area element go to zero gives the value of $\vec{D}$ at $(x, y)$. A problem with this definition is that since there are only finitely many source-destination pairs, and hence only finitely many paths, $\vec{D}$ will be 0 except on a set of measure zero. How this issue is resolved is described below.

We will continue the discussion of the load density vector field temporarily focusing on the special case where the path for each source-destination pair is the line of sight path between the source and destination. In this case $\vec{D}$ is obtained by adding up the weights of all source-destination pairs whose

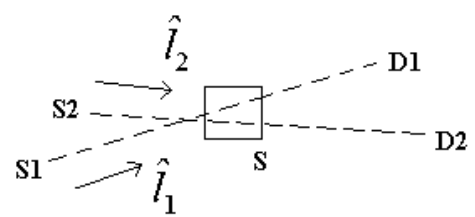

Fig. 1. The illustration of defining the load density vector field based on the line of sight.

line of sight passes through a geographic set in the network and divide it by the area of that set. In other words:

$$
\vec{D}=\lim _{|S| \rightarrow 0} \frac{1}{|S|} \sum_{L_{i} \cap S \neq \emptyset} W_{i} \hat{l}_{i}
$$

in which $S$ is a connected area in the network, $L_{i}$ is a set representing the points residing in the straight line connecting the $i^{\text {th }}$ source-destination pair, and $\hat{l}_{i}$ is a unit vector in the direction of the line of sight of the $i^{t h}$ source-destination pair pointing toward the destination. The above definition has been illustrated in Figure 1 . Note that $\vec{D}$ is a function of the position.

The definition of $\vec{D}$ given by equation (1) satisfies the following equation:

$$
\oint_{C} \vec{D} \cdot \overrightarrow{d n}=w
$$

in which the integral is over a closed contour $C, \overrightarrow{d n}$ is a differential vector normal to the contour at each point of its boundary, dot represents the inner product of vectors in two dimensional space, and $w$ is the algebraic sum of the weights of the sources and destinations inside the closed contour. In calculating $w$ we count the weights of the sources with a positive sign and the weights of the destinations with a negative sign. Equation (2) is analogous to Gauss's law in the electrostatic theory.

Now we define another variable that represents the density of sources in the network and denote it by $\rho$. In other words:

$$
\rho=\lim _{|S| \rightarrow 0} \frac{1}{|S|} \sum_{u_{i} \in S} \operatorname{sgn}\left(u_{i}\right) W_{i}
$$

in which $u_{i}$ is either a source or a destination with weight $W_{i}$, and $\operatorname{sgn}\left(u_{i}\right)$ is 1 if $u_{i}$ is a source and -1 if $u_{i}$ is a destination. With this definition equation (2) can be expressed in partial differential equation form:

$$
\vec{\nabla} \cdot \vec{D}=\rho
$$

where $\vec{\nabla}$ is defined as:

$$
\vec{\nabla}=\frac{\partial}{\partial x} \hat{i}+\frac{\partial}{\partial y} \hat{j}
$$

in which $x$ and $y$ represent the variables in the Cartesian coordinate frames, and $\hat{i}$ and $\hat{j}$ represent the unit vectors along $x$ and $y$ axes respectively.

Mathematically, if the number of source-destination pairs is finite, the values of $\vec{D}$ and $\rho$ defined by equations (1) and (3) will be zero except for a set of measure 0 . In practice we do not need to have large a number of source-destination pairs; we can define a small enough lower bound on the value of $S$ depending on the required accuracy of defining $\vec{D}$. For example, the network terrain can be divided into small rectangles via vertical and horizontal grids, and $S$ can be 


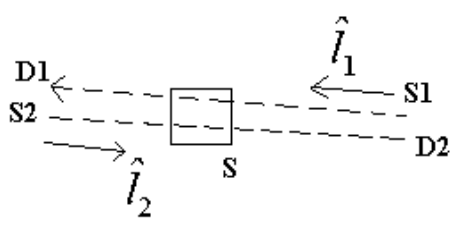

Fig. 2. A situation in which cancellation happens; in this case the value of $\vec{D}$ does not represent the actual value of load in the network

defined as any of these rectangles. In this example the accuracy of $\rho$ and $\vec{D}$ depends on the size of rectangles, and the value of these variables will be constant on each rectangle, so we deal with a discrete version of equations and operators. For example the partial derivative in $x$ direction will be written in terms of the difference of the value of $\vec{D}$ on adjacent horizontal rectangles and the distance between the rectangles. For the sake of simplicity, in the rest of this paper we assume that the size of $S$ is small enough so that we can deal with $\rho$ and $\vec{D}$ as continuous variables.

A problem of the above formulation and definition of $\vec{D}$ is that the amount of load obtained from equation (1) may not reflect the actual need for communication resources in the network. Consider a situation in which we have two sourcedestination pairs that their line of sight intersect with each other but they are heading in opposite directions. In this case the opposite loads might cancel out each other. This situation has been illustrated in Figure 2. This issue may be resolved by partitioning the set of source-destination pairs into groups and putting the cancelling pair in different groups. Each group has its own load vector field. We will see how we can deal with this issue in the next section.

\section{UNIFORM LOAD DISTRIBUTION AND ROUTING}

Given a set of desired paths for each source-destination pair, we obtain a load density vector field $\vec{D}$ as explained in the preceding section. In the special case where the desired paths follow line of sight, $\vec{D}$ satisfies the following equations

$$
\left\{\begin{array}{l}
\vec{\nabla} \cdot \vec{D}=\rho \\
D_{n}(z)=0 \text { for } z \in \text { Boundary of } A
\end{array}\right.
$$

in which $A$ denotes the geographical set that contains the network and $D_{n}(z)$ denotes the normal component of $\vec{D}$ on the boundary of $A$. The first equation in (6) is the natural limitation imposed by the sources and the destination. The second equation comes from the fact that no load is desired to exit the geographical area of the network or enter into it through the boundary.

It is straightforward to show that for any load density vector field (not necessarily line of sight), equations (6) are satisfied. In fact, an alternative definition of a load density vector field is any vector field satisfying these equations. The original definition $\vec{D}$ in the preceding section started with a set of desired paths and obtained from that the vector field. Using the alternative definition, we will be able to generate a set of desirable paths by imposing conditions that together with (6) uniquely specify $\vec{D}$, and then deriving the paths from $\vec{D}$.

We approach the problem in two steps. First we consider a many-to-one communication scenario. Then we apply the results of the many-to-one case scenario to the general case in which every two arbitrary nodes can communicate with each other.

\section{A. Many-to-One Scenario}

In this scenario there is only one destination in the network with which all sources communicate. A practical example of a many-to-one scenario is the case in which the nodes in a big network are divided into many clusters, and each cluster has a clusterhead [13]. Each node in a cluster needs to send its data to the clusterhead in order to communicate with nodes in other clusters. The communication directed from the cluster nodes to their clusterhead is an example of many-toone communication.

In the many-to-one scenario, if we have a $\vec{D}$ that satisfies equations (6), we can find the routes that can be used to send the traffic of sources to the destination. In order to define the routes based on the values of $\vec{D}$, we need to define the concept of load flow lines. These lines are similar to the electric flux lines in electrostatic theory [1] [2]. The load flow lines are a family of two dimensional curved lines that are always tangent to the direction of the $\vec{D}$ and their orientation is the same as the orientation of the $\vec{D}$. The load flow lines cannot intersect except at a source or the destination; if they intersect, at the point of intersection the direction of the field would not be single-valued. The other property of the load flow lines is that these lines always start at the sources and end at the destination; this fact is because the value of divergence in equations (6) is positive at the sources, and it is negative only at the destination.

We use the concept of the load flow lines to derive routing from $\vec{D}$. If we are at a source like $S_{i}$, we can start a short range transmission along the direction of one of the load flow lines going out of this source. We assume the nodes are densely distributed in the geographical area of the network, such that a node like node $R_{1}$ exists such that the line connecting $S_{i}$ to $R_{1}$ is close enough to the load flow line. Node $R_{1}$ continues relaying the packet of $S_{i}$ by sending it to another node line $R_{2}$ in the direction of the load flow line. By using this mechanism and considering the fact that the load flow lines always end at the destination we are guaranteed to send the packets of the sources to the destination. This scheme has been illustrated in Figure 3. In this figure the solid curve shows the direction of a typical load flow line. As it has been shown, this load flow line has been approximated by a sequence of wireless links between nodes.

One issue that must be resolved regarding the above approach for routing is that theoretically it might happen that infinitely many load flow lines might start from each source, and we should solve the problem of which load flow lines should be chosen for the purpose of routing. If we can create $P$ paths from the source to the destination, we propose to pick $P$ load flow lines that start at the source and depart from the source in different and evenly spaced directions. For example if $P=8$, the angle between successive load flow lines at the source is $360^{\circ} / 8=45^{\circ}$. In this example the source breaks its traffic among 8 paths. The amount of traffic assigned to each path is proportional to the magnitude of the $\vec{D}$ at the source along load flow line corresponding to that path. Based 


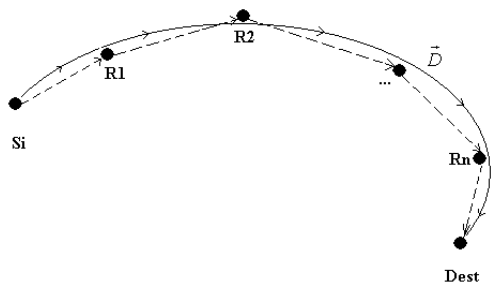

Fig. 3. Approximating the path given by a load flow line by the links made by the relaying nodes.

on the fact that there is only one destination in the network for the many-to-one scenario, the cancellation problem that we discussed in the previous section does not exist for this case.

So far we have established the basic concept of load vector field, and described its connection to routing in the many-toone scenario. Thus, given $\vec{D}$, we can obtain routes. However, equations (6) do not specify $\vec{D}$ uniquely. The remaining issue is to decide what additional condition(s) to place on $\vec{D}$ so the resulting vector field generates a desirable set of routes. The intuition we follow is that by making $\vec{D}$ as uniform as possible, we will obtain routes that will cause the traffic to be highly dispersed throughout the network. In turn, this will decrease both node congestion and collisions and lead to high throughput.

The uniform load distribution can be formulated as minimizing the following cost function:

$$
J(\vec{D})=\int_{A}\left|\left(\vec{D}-\vec{D}_{a v}\right)\right|^{2} \mathrm{ds}
$$

in which $\overrightarrow{X_{a v}}$ is the average value of the vector field $\vec{X}$ on the set $A$, and it can simply defined as:

$$
\vec{X}_{a v}=\frac{1}{|A|} \int_{A} \vec{X} \mathrm{ds} .
$$

The quadratic form of the cost function in equation (7) causes the load to be distributed as uniformly as possible. It prevents having high loads somewhere in the network while the resources are underutilized somewhere else. One interesting fact about this cost function is that it is similar to the definition of energy in electrostatic theory. The above optimization problem can be summarized as:

$$
\begin{aligned}
& \text { Minimize } J(\vec{D})=\int_{A}\left|\left(\vec{D}-\vec{D}_{a v}\right)\right|^{2} \mathrm{ds} \\
& \text { Subject to: } \\
& \vec{\nabla} \cdot \vec{D}=\rho \\
& D_{n}(z)=0 \quad z \in \text { Boundary of } A
\end{aligned}
$$

The following lemma provides the key to finding the solution of the optimization problem defined by (9).

Lemma 1: If $\overrightarrow{D^{*}}$ denotes the optimal solution of equation (9), then it satisfies:

$$
\vec{\nabla} \times \overrightarrow{D^{*}}=0
$$

In the above equation $\vec{\nabla} \times$ is the two dimensional curl operator, and it is defined in the following way for a vector field $\vec{F}=$ $\left[\begin{array}{ll}F_{x} & F_{y}\end{array}\right]$ :

$$
\vec{\nabla} \times \vec{F}=\left(-\frac{\partial F_{x}}{\partial y}+\frac{\partial F_{y}}{\partial x}\right) \hat{k}
$$

in which $\hat{k}$ is a unit vector perpendicular to $\hat{i}$ and $\hat{j}$. More precisely, $\hat{k}=\hat{i} \times \hat{j}$. The proof of Lemma 1 is given in [10].
Based on the result of the this lemma, we can write a set of partial differential equations for the optimal $\overrightarrow{D^{*}}$ :

$$
\vec{\nabla} \cdot \overrightarrow{D^{*}}=\rho \quad \vec{\nabla} \times \overrightarrow{D^{*}}=0
$$

The above equations are similar to Maxwell's equations in the electrostatic theory. In the theory of partial differential equations it is proved that the above equations along with the boundary condition given by (6) give $\overrightarrow{D^{*}}$ uniquely. The interesting fact is that in the theory of electrostatics, Maxwell's equations imply that the stored energy in the space is minimal. However, our definition of energy is a little bit different from that in the electrostatic theory.

Mathematically, a vector field for which $\vec{\nabla} \times \vec{D}=0$ is called a conservative vector field. It is proved that such a vector filed can be expressed as the gradient of a scalar field. In other words:

$$
\vec{D}=\vec{\nabla} U
$$

in which $U$ is a scalar function known as the potential function. Then the set of equations defined by (12) reduces to:

$$
\nabla^{2} U=\rho
$$

in which the operator $\nabla^{2}$ is defined as:

$$
\nabla^{2}=\frac{\partial^{2}}{\partial x^{2}}+\frac{\partial^{2}}{\partial y^{2}}
$$

The boundary conditions for $\vec{D}$ implies that the gradient of $U$ is zero on the boundary along the direction that is normal to the boundary. In other words:

$$
\vec{\nabla} U(z) \cdot \hat{n}(z)=0 \quad z \in \text { Boundary of } A
$$

in which $\hat{n}(z)$ is a unit vector normal to the boundary.

The partial differential equation defined by (14) is known as the Poisson equation. The potential function found as the solution of this equation has very nice interpretations for the many-to-one scenario. Firstly, the potential function gives a rough idea of how much effort by the network is needed to send data from a source to the destination. This effort is proportional to the potential difference of the source and the destination. Secondly, the potential function gives insight into the routing. Based on equation (13), the routing is done in the direction of the gradient of the potential function. Some concerns like the possibility of forming routing loops are naturally avoided since the potential function changes strictly monotonic in the nodes that form a path from the source to the destination.

\section{B. General Case}

Now we turn our attention to the general case in which every arbitrary pair of nodes can communicate with each other. Our approach to this problem is to break it into several manyto-one problems. We consider the case in which there are several destinations, and all sources want to communicate to these destinations. Assume there are $m$ such destinations in the network denoted by $\Omega=\left\{d_{1}, d_{2}, \ldots, d_{m}\right\}$.

Now we can formulate the problem of uniform resource utilization in the general case. Assume $\vec{D}_{i}$ and $\rho_{i}$ denote the 
load density vector field and density of sources for the $i^{t h}$ destination respectively. We have:

$$
\vec{\nabla} \cdot \vec{D}_{i}=\rho_{i}
$$

It follows from the original definition of the load density vector field as being derived from the tangent vectors of the desired paths for each source-destination pair that $\vec{D}=\sum_{i=1}^{m} \vec{D}_{i}$. To fully determine $\vec{D}$, we need to specify the optimization problem that it should satisfy. One way of generalizing the uniform load distribution problem is to formulate it as the following optimization problem:

$$
\begin{aligned}
& \text { Minimize } J=\sum_{i=1}^{m} \int_{A}\left|\vec{D}_{i}-\vec{D}_{i a v}\right|^{2} \mathrm{ds} \\
& \text { Subject to: } \\
& \vec{\nabla} \cdot \vec{D}_{i}=\rho_{i} \quad 1 \leq i \leq m \\
& D_{n i}(z)=0 \quad z \in \text { Boundary of } A \quad 1 \leq i \leq m
\end{aligned}
$$

in which $D_{n i}(z)$ denotes the normal component of $D_{i}$ on the boundary of $A$, and $\vec{D}_{i a v}$ denotes the average of $\vec{D}_{i}$ on $A$ as it is defined by equation (8). The above formulation of the cost function implies that the problem is decomposed into several optimization subproblems, and for the $i^{t h}$ destination we have:

$$
\begin{aligned}
& \text { Minimize } J_{i}=\int_{A}\left|\vec{D}_{i}-\vec{D}_{i a v}\right|^{2} \mathrm{ds} \\
& \text { Subject to: } \\
& \vec{\nabla} \cdot \vec{D}_{i}=\rho_{i} \\
& D_{n i}(z)=0 \quad z \in \text { Boundary of } A
\end{aligned}
$$

or equivalently:

$$
\begin{aligned}
& \vec{\nabla} \cdot \vec{D}_{i}=\rho_{i} \quad \vec{\nabla} \times \vec{D}_{i}=0 \\
& D_{n i}(z)=0 \quad \text { for } z \in \text { Boundary of } A
\end{aligned}
$$

The form of the cost function in equation (18) does not ensure an integrated optimization for different destinations since the value of the load density vector fields for different destinations are obtained independently. One might make changes in the form of the cost function to achieve an integrated optimal solution. One way to do this is to change the cost function in the following way to achieve an integrated solution:

$$
J=\int_{A}\left(\sum_{i=1}^{m}\left|\vec{D}_{i}-\vec{D}_{i a v}\right|\right)^{2} \mathrm{ds}
$$

This cost function is consistent with the definition of the cost in the case of $m=1$; however, it is not quadratic except for $m=$ 1. Unfortunately, it is hard to find simple partial equations that give the solution of the problem when the cost function is defined by equation (21), but this optimization problem can be solved by using numerical methods.

\section{Simulation Results}

In this section we will show the results of the simulation for the proposed method of distributing the load in the network. In this simulation scenario nodes of the network are distributed in a $1000 m \times 1000 m$ square. The number of nodes in the experiment is 1500 . We have simulated a many-to-one scenario in which all sources try to send their data toward a single destination. In this experiment we have assumed the destination is located in the center of the square. The number of sources is 40 and the sources are randomly distributed in

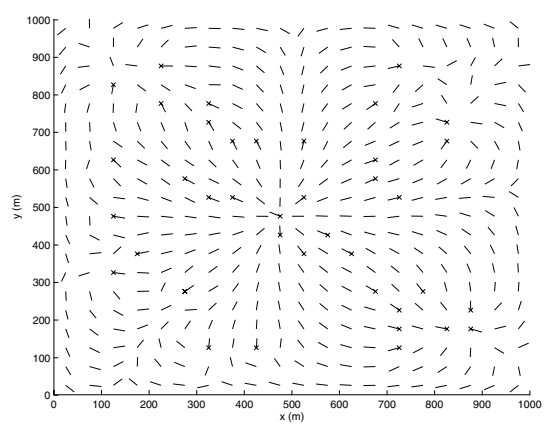

Fig. 4. The placement of sources and the direction of optimal $\vec{D}$ : each $\times$ shows a source, and the line segments show the direction of the optimal $\vec{D}$.

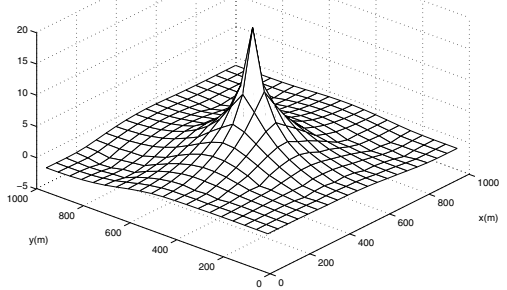

Fig. 5. The value of the potential function $U$

the network, and they have equal weights.

Figure 4 shows the direction of the $\vec{D}$ for the given arrangement of sources. In this figure each $\times$ represents a source, except the $\times$ in the center in the coordinate $(500 \mathrm{~m}, 500 \mathrm{~m})$ that shows the destination. We have numerically solved the Poisson equation given by equation (14) with the boundary condition given by equation (16) on a $20 \times 20$ grid to find the potential function $U$. Then we have found $\vec{D}$ by taking the gradient of $U$. The line segments in this figure show the direction of the optimal load density vector field $\vec{D}$ in the network terrain. As it can be seen in this figure, the optimal $\vec{D}$ diverges from the sources and converges to the destination in the middle. The other observation about this figure is that the direction of $\vec{D}$ deviates from the line of sight at the places of the network that are far enough from the destination, and it gets closer to the line of sight of the destination in the vicinity of it.

In the experiment, we have split the demand of each source among 8 paths that go out from that source in evenly spaced directions. In other words, the traffic from each source uses multipath with 8 paths from the source to the destination. The amount of load assigned to each path is proportional to the absolute value of the $\vec{D}$ on that direction. It should be emphasized that the splitting is done only at the sources, and once the traffic of the source is split, it follows the direction of optimal $\vec{D}$ toward the destination. Figure 6 shows the routes found by this method. In the figure each $\diamond$ represents a source. As can be seen in the figure, eight routes are used for each source. Figure 5 shows the value of potential function $U$ found by numerically solving equation (14) on a $20 \times 20$ grid.

To have a basis of comparison we have also calculated the routes that use the line of sight. In this routing scheme each source tries to send its data toward the destination by relaying its data to the node that is within its communication range and is closest to the destination. Figure 7 shows the routes calculated by this method. By comparing this figure 


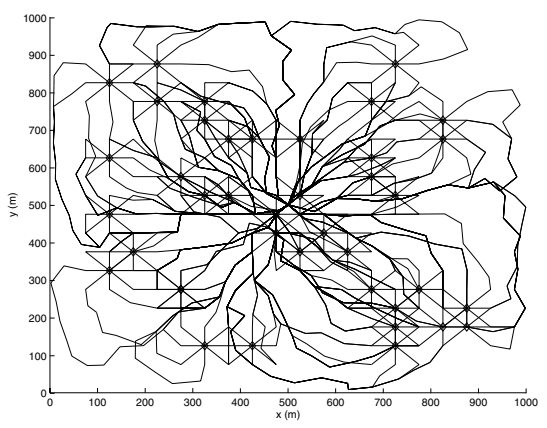

Fig. 6. The routes from the sources to the destination. Each $\diamond$ represents a source; eight routes are assigned to each source.

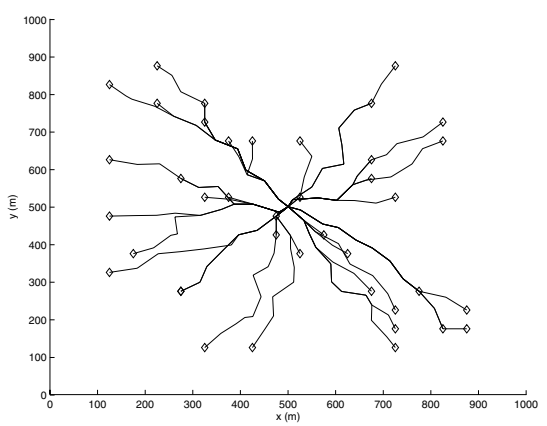

Fig. 7. The routes from the sources to the destination. Each $\diamond$ represents a source; in this case routes from each source to each destination are close to their line of sight.

with figure 6 , it can be seen that in the case of using optimal $\vec{D}$ the resources of the network are utilized much more uniformly. To evaluate the difference of using optimal $\vec{D}$ to the case in which we use the line of sight, we have simulated both cases by using Qualnet [12] simulator. In the simulation experiments the MAC protocol is 802.11, and the maximum transmission range of each node is about 50 meters. Each source is considered as CBR source sending blocks of 256 bytes in the intervals of 125 milliseconds toward the destination. The simulation results show that the total throughout of the network in the case of using optimal distribution of load is about $5.6 \times 10^{5} \mathrm{bits} / \mathrm{s}$ while this value is $3.3 \times 10^{5} \mathrm{bits} / \mathrm{s}$ for the line of sight approach. Thus we have gained about a 70 percent increase by optimal distribution of the load in the network.

We have done several other experiments with the same conditions as the above experiment but with different randomly generated locations of the nodes and the traffic sources. The results are shown in Table I. The second column of this table shows the throughput achieved by the electrostatic approach in bits/s, and the third column shows the corresponding values for the line of sight case. It can be seen that in all cases the throughput is increased considerably, and the average increase is $34 \%$.

\section{CONClusions ANd Future Works}

In this paper we introduced an approach to the routing problem in wireless ad hoc networks. We assumed that the routing between a source and destination pair can be done in a multihop and multipath way. We made the conjecture that a better performance of the network in terms of throughput can be achieved if we try to distribute the communication load as

\begin{tabular}{|c|c|c|c|}
\hline Exp. & Elec. & Line of Sight & improvement \\
\hline 1 & $6.5 \times 10^{5}$ & $4.6 \times 10^{5}$ & $39 \%$ \\
\hline 2 & $5.5 \times 10^{5}$ & $3.8 \times 10^{5}$ & $43 \%$ \\
\hline 3 & $5.7 \times 10^{5}$ & $5.0 \times 10^{5}$ & $14 \%$ \\
\hline 4 & $5.4 \times 10^{5}$ & $3.6 \times 10^{5}$ & $51 \%$ \\
\hline 5 & $5.8 \times 10^{5}$ & $4.7 \times 10^{5}$ & $23 \%$ \\
\hline
\end{tabular}

TABLE I

The comparison of the electrostatic approach with the line of sight approach for different simulation runs

uniformly as possible across the network. Under this assumption, in the case that many sources want to send their data to a single destination, the optimal solution of the problem can be achieved by solving a set of partial differential equations similar to Maxwell's equations in electrostatic theory.

In this work we have assumed that the information about the location of the nodes and the amount of the demand of sources is known in a central route server. However, a decentralized approach can be obtained by using a flooding mechanism in which the nodes advertise the information of their location and their demands to to the other nodes in the network. Another direction for extending our work is taking into account the possible mobility of the sources. In this case the load vector field will be time varying, and it might be possible to make an analogy between the routing problem and the electric field propagation in the electrodynamics theory.

\section{ACKNOWLEDGMENT}

We thank the Qualnet University Program for providing the Qualnet software.

\section{REFERENCES}

[1] McQuistan, B. R., "Scalar and Vector Fields: A Physical Interpretation," John Wiley \& Sons, 1965.

[2] Jackson , J. D., "Classical Electrodynamics," Third Edition, John Wiley \& Sons, 1999.

[3] Matthews, P.C., "Vector Calculus," Springer 1998.

[4] Hong, X., Xu, K., and Gerla, M., "Scalable Routing Protocols for Mobile Ad Hoc Networks," IEEE Network Magazine, July-Aug, 2002, pp. 11-21.

[5] Huitema, C., "Routing in the Internet," Second Edition, Prentice Hall PTR, 1999.

[6] Iwata, A., Chiang, C., Pei, G., Gerla, M. and Chen, T., "Scalable Routing Strategies for Ad hoc Wireless Networks," IEEE JSAC, Aug. 1999, pp 1369-79.

[7] Pei, G., Gerla, M., and Chen, T., "Fisheye State Routing: A Routing Scheme for Ad Hoc Wireless Networks," Proc. of ICC, June 2000.

[8] Perkins, C. E., and Royer, E. M., "Ad Hoc On-Demand Distance Vector Routing," Proc. of IEEE WMCSA, Feb 1999.

[9] Ko, Y. B. and Vaidya N. H., "Location-aided Routing (LARE) in Mobile Ad Hoc Networks," Proc. of MOBICOM, October, 1998.

[10] Kalantari, M. and Shayman M., "Electrostatic Routing in Ad Hoc networks," The Institure for Systems Research Technical Report, available online at http://techreports.isr.umd.edu/ARCHIVE/.

[11] Basagni, S., Chlamtac, I., Syrotiuk, V. R., and Woodward, B., "A Distance Routing Effect Algorithm for Mobility (DREAM)," Proc. of MOBICOM, October 1998.

[12] Qualnet, online information available at: http://www.scalable-networks.com/.

[13] Chiang, C. and Gerla, M., "Routing and Multicast in Multihop, Mobile Wireless Networks,” Proc. of IEEE ICUPC, Oct. 1997. 